\title{
ARTICLE
}

\section{Varacin-1, a novel analog of varacin C, induces p53- independent apoptosis in cancer cells through ROS-mediated reduction of XIAP}

\author{
Jing Zhou ${ }^{1}$, Wen-li Li ${ }^{2}$, Zi-xuan Wang ${ }^{3,4}$, Nai-yuan Chen ${ }^{4}$, Yue Tang ${ }^{3,4}$, Xiao-xiao Hu ${ }^{3,4}$, Jing-huan Deng ${ }^{4}$, Yixin Lu Lu $^{5}$ Guo-dong Lu ${ }^{3,4,6}$
}

\begin{abstract}
Varacin $C$ is a promising anticancer agent and possesses acid-promoted and photo-induced DNA-damaging activities. In this study, we synthesized an analog varacin-1 (VCA-1) and examined its anticancer potentials. The results demonstrated that VCA-1 caused dose-dependent apoptotic cell death in cancer cells. Note that this action is independent of p53 status, because VCA-1 induced similar levels of apoptosis in two different panels of cell lines (HCT116 p53- wild-type vs. HCT116 p53-knockout colon cancer cells, and p53-expressing U2OS vs. p53-deficient saos2 osteosarcoma cancer cells). VCA-1-induced apoptosis was found to be mainly via the extrinsic apoptosis pathway involving caspase-8 activation and XIAP reduction. Forced over-expression of XIAP markedly prevented apoptosis, indicating its essential role in VCA-1 induced apoptosis. On the other hand, VCA-1 treatment enhanced intracellular ROS (reactive oxygen species) generation also in a p53-independent manner, and consequently promoted caspase activation. Pretreatment of N-acetyl cysteine (an antioxidant), rather than z-VAD (specific caspase inhibitor), markedly prevented XIAP reduction, suggesting that XIAP reduction may be resulted from oxidative stress. In conclusion, data from this study reveal the essential roles of ROS generation and XIAP reduction in VCA-1-induced apoptosis in cancer cells. VCA-1 may be a novel cancer therapeutic agent, especially in p53-mutant human cancers.
\end{abstract}

Keywords: varacin-1; apoptosis; p53; XIAP; ROS

Acta Pharmacologica Sinica (2019) 40:222-230; https://doi.org/10.1038/s41401-018-0005-y

\section{INTRODUCTION}

Varacin, as a bicyclic organosulfur compound, was first discovered from the Far Eastern ascidian Polycitor sp. in 1995 [1]. Unlike varacin and any other previously discovered microbial metabolites, the newly-synthesized varacin $C$ possesses a unique trithiol 1-oxide as its reactive functionality [1]. Since varacin $C$ is the only anticancer agent discovered to date having such a major functionality, it is tempting to develop a group of molecules with similar structures to varacin C. The cytotoxic effect of varacin C was found to be related to its DNA-damaging activity and essentially dependent on an oxymethyl substituting group at the second position of the aromatic ring [2]. Note that the DNA-cleaving properties of varacin $C$ exhibit an acid-promoted and photoinduced pattern [3]. It is thus possible to specifically excite the cytotoxicity of varacin $C$ in the cancer cells which usually have acidic extracellular microenvironment and intracellular organelles compared to normal cells $[4,5]$. The photosensitizing property also enables varacin $C$ to be useful particularly in photodynamic chemotherapy. The investigation of varacin $C$ and its analogs on their potential anticancer properties and underlying molecular mechanisms might lead to the discovery of novel anticancer drugs.

It has been well-established that p53 tumor suppressor is the main regulator for DNA damage and apoptosis [6-8]. However, p53 is mutated or deficient in almost $50 \%$ of human tumors $[9,10]$. The mutated p53 could even promote cell invasion and tumor metastasis $[11,12]$. Thus, it would be of great interest to find a chemotherapeutic approach to target p53-mutant or null tumor. In this study, we synthesized varacin-1 (VCA-1) as an analog of varacin $C$ and evaluated whether VCA-1 could potently induce apoptosis in p53-negative tumor cells. We further evaluated the involvement of reactive oxygen species (ROS) in VCA-1-induced apoptosis, because varacin C's cytotoxicity was found to be reliant on the induced oxidative stress and consequent DNA damage [3]. Data from this study provided sufficient experimental evidence to support the potential anticancer property of VCA-1.

\footnotetext{
${ }^{1}$ Department of Physiology, School of Preclinical Medicine, Guangxi Medical University, Nanning 530021, China; ${ }^{2}$ Guangdong Provincial Center for Disease Control and

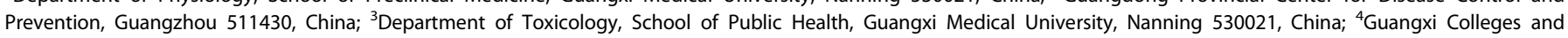

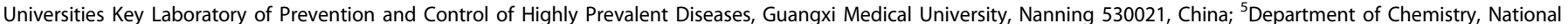

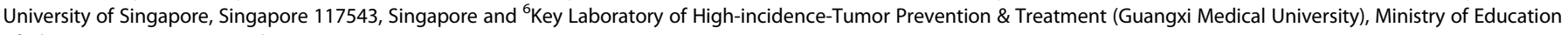
of China, Nanning 530021, China

Correspondence: Guo-dong Lu (golden_lu@hotmail.com)
}

These authors contributed equally: Jing Zhou, Wen-li Li.

Received: 13 October 2017 Revised: 16 January 2018 Accepted: 16 January 2018

Published online: 17 May 2018 


\section{MATERIALS AND METHODS}

Synthesis of VCA-1

VCA-1 was synthesized from vanillin following ten-step procedures in Lu Y's lab in the Department of Chemistry in the National University of Singapore. Briefly, vanillin was converted to $\mathrm{N}$-(Boc)-4-methylamino-6,7-dimethoxy-1,2,3-benzotrithiole 2-oxide through a series of reactions. After irradiation with wavelength of $300 \mathrm{~nm}$ for $4 \mathrm{~h}, \mathrm{~N}$-(Boc)-4-methylamino-6,7dimethoxy-1,2,3-benzotrithiole 3-oxide was purified and then reacted with trifluoroacetic acid to form 4-methylamino-6,7dimethoxy-1,2,3-benzotrithiole 3-oxide trifluoroacetate (VCA-1). DNA cleavage assay was conducted at $37^{\circ} \mathrm{C}$ for $12 \mathrm{~h}$ in $50 \mathrm{mM}$ sodium phosphate ( $\mathrm{pH}$ 5.9) with increasing dose of each analog and $0.5 \mu \mathrm{g}$ supercoiled pBR322 DNA as substrate. The band densities of the full-size pBR322 DNA were quantified with ImageJ software (version 1.51q) to calculate $\mathrm{EC}_{50}$ (half maximal effective concentration) of the respective DNA cleavage activity.

Reagents and antibodies

Hoechst and 5-(and-6)chloromethyl-2', $7^{\prime}$-dichlorodihydrofluorescein diacetate acetyl ester (CM- $\mathrm{H}_{2}$ DCFDA) were purchased from Invitrogen (Molecular Probes, Eugene, OR, USA). N-acetyl cysteine (NAC) was from Sigma (St Louis, MO, USA). Pan-caspase inhibitor z-VAD-FMK was purchased from BioMol (Plymouth Meeting, PA, USA). Anticaspase-9, anticaspase-8, anticaspase-3 and anti-poly (ADP-ribose) polymerase antibodies were from Cell Signaling (Beverly, MA, USA). Anti-XIAP antibody was from BD Transduction Laboratories (San Diego, CA, USA). Anti-tubulin and anti-actin antibodies, caspase 8 specific inhibitor z-IETD-FMK and caspase 9 specific inhibitor z-LEHD-FMK were obtained from Santa Cruz Biotechnology (Santa Cruz, CA, USA).

Cell culture

Human HCT116 p53-WT/KO colorectal cancer cells were kind gifts from Dr. Vogelstein (Johns Hopkins University, Baltimore, MA, USA) and human U2OS/Saos2 osteosarcoma p53-WT/KO cancer cells were purchased from American Type Culture Collection (Manassas, VA, USA). Cells were maintained in Dulbecco's modified Eagle's medium supplemented with $10 \%$ fetal bovine serum (FBS; Hyclone, Logan, UT) in a $5 \% \mathrm{CO}_{2}$ atmosphere at $37^{\circ} \mathrm{C}$. Equal amounts of cells were seeded and switched to the medium supplemented with $1 \%$ FBS before treatment. The same concentration of DMSO was always applied to control cells. All the inhibitors were added into the wells $1 \mathrm{~h}$ before VCA-1 treatment.

\section{Detection of apoptosis}

Cell death was determined by several approaches quantitatively and qualitatively, as described previously $[13,14]$. First, morphological changes under phase-contrast microscopy were employed. Second, Hoechst staining was used to examine chromatin condensation, which is characteristic for cell death. Briefly, after designated treatments, cells were stained with $10 \mu \mathrm{g} / \mathrm{ml}$ Hoechst for $30 \mathrm{~min}$ at $37^{\circ} \mathrm{C}$. Cell nuclei were visualized under an inverted fluorescence microscope and photographed (Nikon ECLIPSE TE2000-S, Nikon Instruments, Tokyo, Japan). At least 200 cells from three separate images were counted to quantitatively determine the proportion of dead cells. The caspase- 3 activities by VCA-1 treatment, together with different caspase inhibitors, were measured by a colorimetric assay kit from Abcam (Cambridge, UK). Briefly, HCT116 p53-WT/KO cells were treated with VCA-1 $(20 \mu \mathrm{M})$ for $24 \mathrm{~h}$, in the absence or presence of different caspase inhibitors (all at $20 \mu \mathrm{M}$ ). The absorbance at $405 \mathrm{~nm}$ was then determined by a spectrophotometer.

Measurement of intracellular $\mathrm{y}-\mathrm{H} 2 \mathrm{AX}$ and ROS

The cellular levels of DNA double-strand break were monitored by $\gamma-\mathrm{H} 2 \mathrm{AX}$ formation (phosphor 139 antibody \#ab11174 from Abcam) a
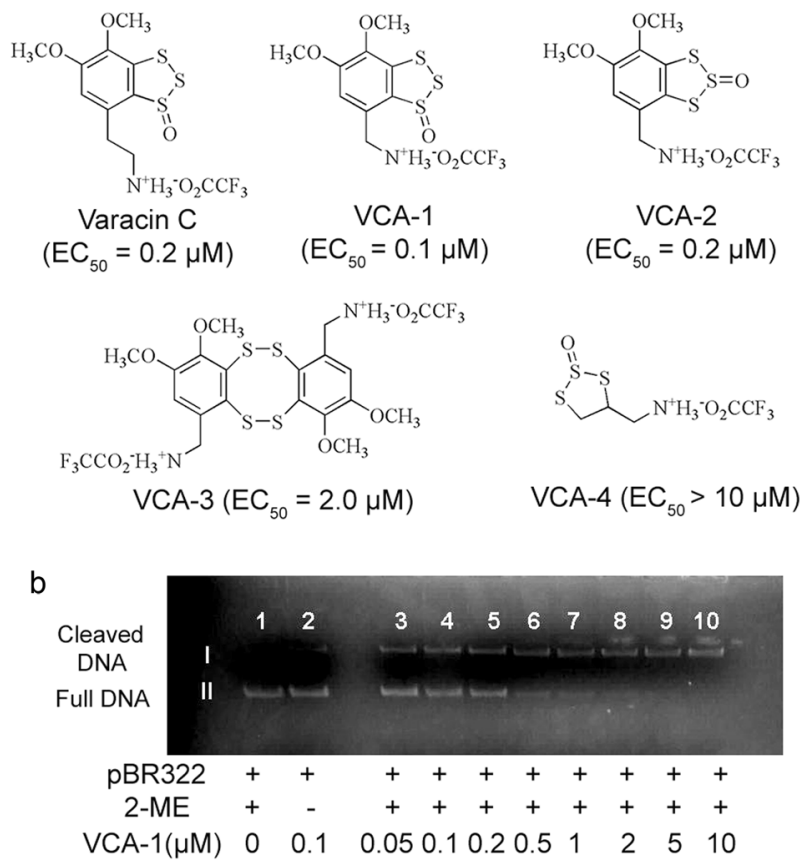

C

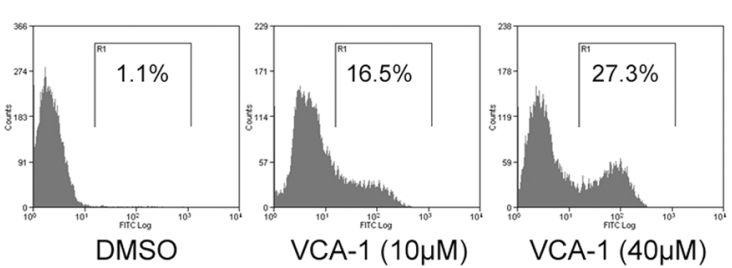

Fig. 1 VCA-1 has the highest DNA-cleaving activity. a Chemical structure of VCA-1 and another four relevant chemicals. b DNA cleavage by various concentration of VCA-1. c The cellular levels of DNA double strand break were monitored by the $\gamma$-H2AX formation assay after the indicated VCA-1 treatment for $12 \mathrm{~h}$

using flow cytometry analysis according to the manufacturer's protocol. The HCT116 cells were treated with VCA-1 for $12 \mathrm{~h}$ before paraformaldehyde fixation and subsequent $y-\mathrm{H} 2 \mathrm{AX}$ and FITC-conjugated secondary antibody staining. Intracellular ROS were measured by $\mathrm{CM}-\mathrm{H}_{2} \mathrm{DCFDA}$ as described previously [14]. Briefly, cells were stained with $\mathrm{CM}-\mathrm{H}_{2}$ DCFDA at $37^{\circ} \mathrm{C}$ for $30 \mathrm{~min}$ after the indicated treatment. Then the cells were collected and analyzed by a BD FACSCalibur ${ }^{\mathrm{TM}}$ flow cytometry (BD Biosciences, San Jose, CA, USA).

Western blotting

At the end of the designated treatments, cells were lysed in $\mathrm{M} 2$ cell lysis buffer (20 mM Tris at pH 7, 0.5\% NP-40, $250 \mathrm{mM} \mathrm{NaCl}, 3$ $\mathrm{mM}$ EDTA, $3 \mathrm{mM}$ EGTA, $2 \mathrm{mM}$ dithiothreitol, $0.5 \mathrm{mM}$ phenylmethylsulfonyl fluoride, $20 \mathrm{mM}$ glycerol phosphate, $1 \mathrm{mM}$ sodium vanadate, and proteinase inhibitor cocktail) [14]. After blocking with StartingBlock ${ }^{\mathrm{TM}}$ (PBS) blocking buffer (Thermo), the membrane was probed with various antibodies and developed with enhanced chemiluminescence (Pierce) using a Kodak Image Station 440CF (Kodak). The band density was quantified with ImageJ software.

Transient transfection

Lipofectamine Plus reagent from Invitrogen (Carlsbad, CA, USA) was used to transfect pcDNA, Flag-XIAP or CrmA into cells 


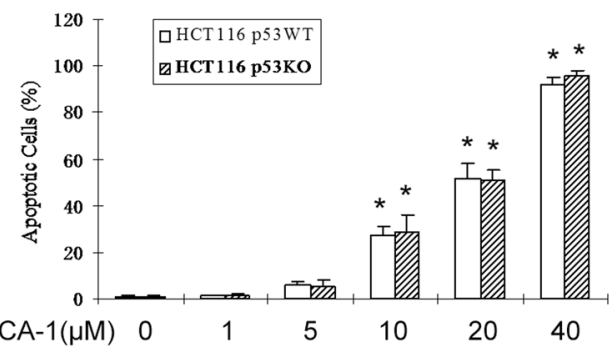

b

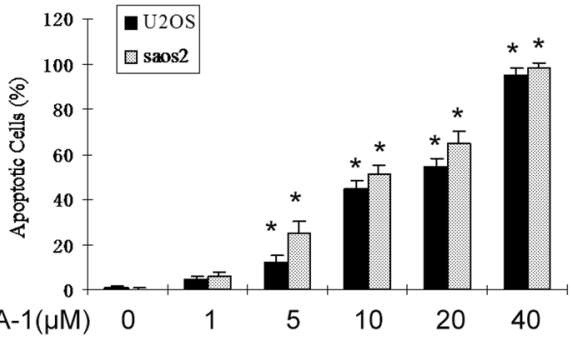

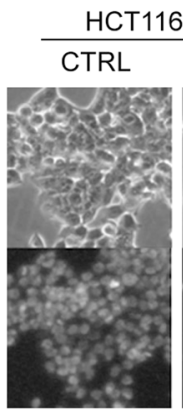
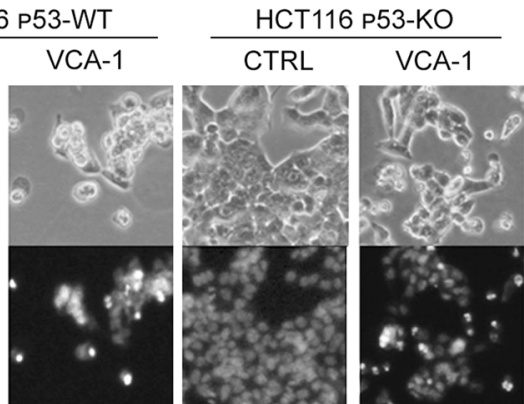

C
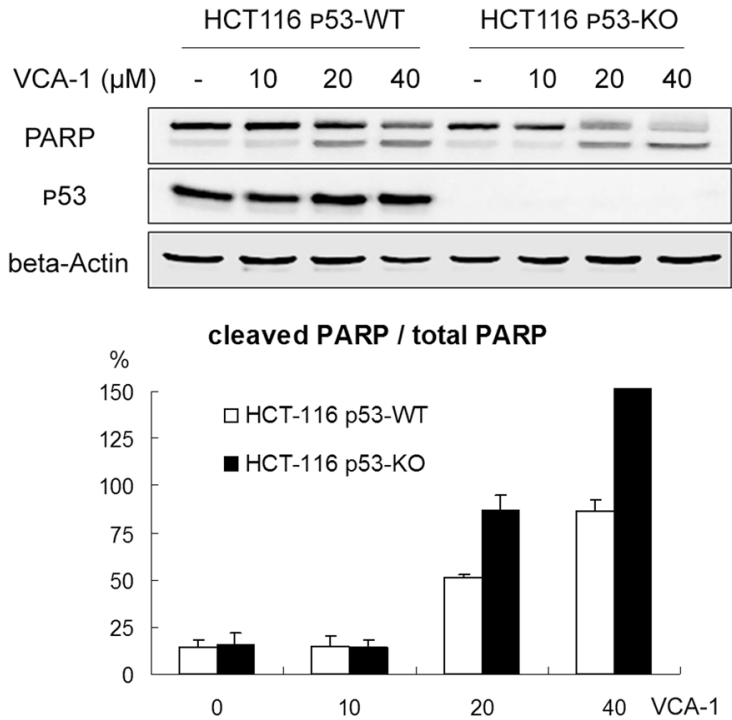

e

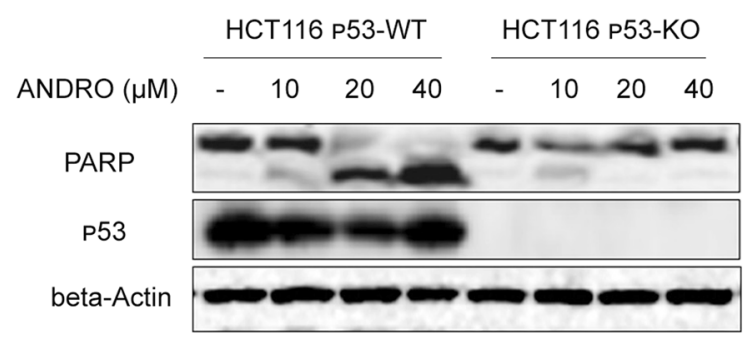

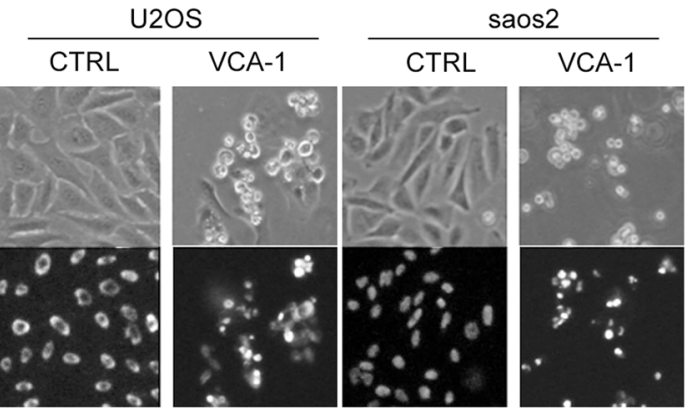

d
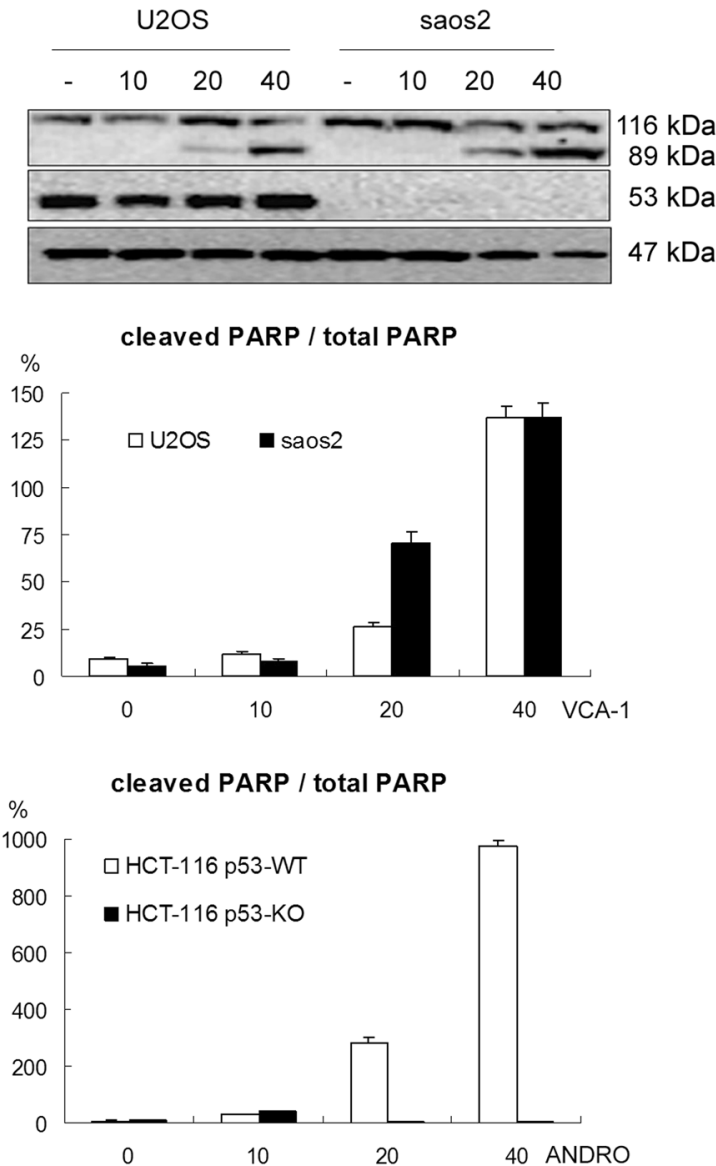

Fig. 2 VCA-1 induces p53-independent apoptosis in human cancer cell lines. a Effects of VCA-1 on HCT116 human cancer cells. Upper panel: dose-dependent apoptosis induced by VCA-1 in HCT116 p53-WT/KO cells. Cells were treated with VCA-1 for indicated doses for $24 \mathrm{~h}$ and cell death was determined using Hoechst staining as described in Materials and Methods. Data were presented as mean \pm SD from three independent experiments. Lower panel: representative images were taken by a phase-contrast microscope $(\times 200)$ after treatment with VCA-1 $(20 \mu \mathrm{M})$ for $24 \mathrm{~h}$. b Effects of VCA-1 on U2OS/Saos2 human cells. Cells were treated as same as described in (a). c, d VCA-1-induced PARP cleavage in human cancer cells. HCT116 p53-WT/KO (c) and U2OS/Saos2 cells (d) were treated with VCA-1 for designated doses for $24 \mathrm{~h}$. After the treatment, cell lysate was prepared and subjected to Western blotting. e Andro-induced PARP cleavage in p53-WT human cancer cells. HCT116 p53-WT/KO cells were treated with Andro for designed doses for $24 \mathrm{~h}$. ${ }^{*} P<0.05$, compared to the untreated control 
a

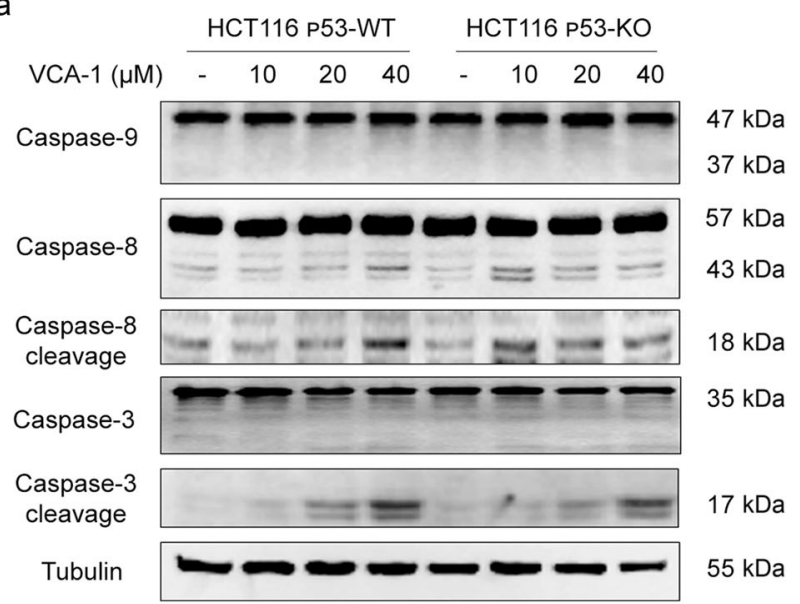

b
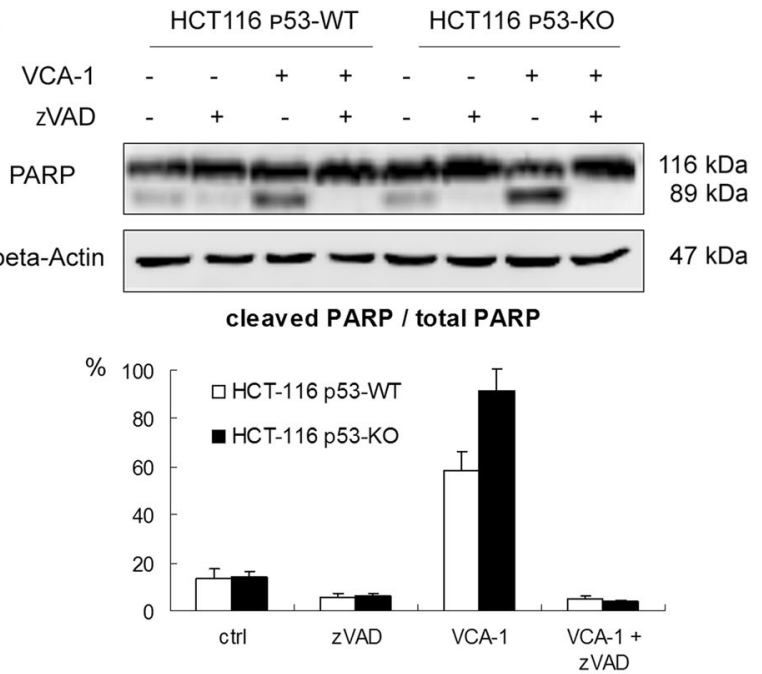

C

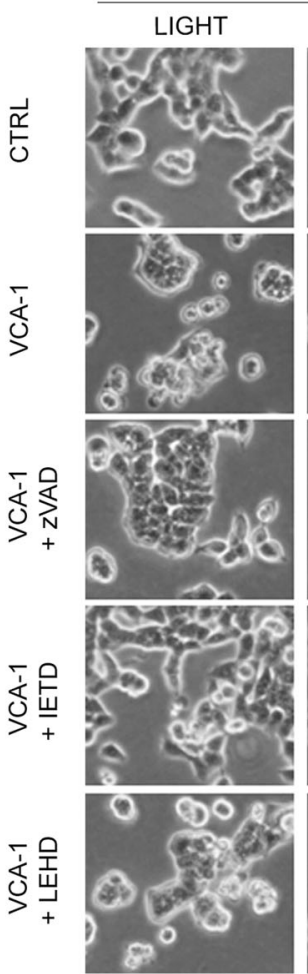

HCT116 P53-KO

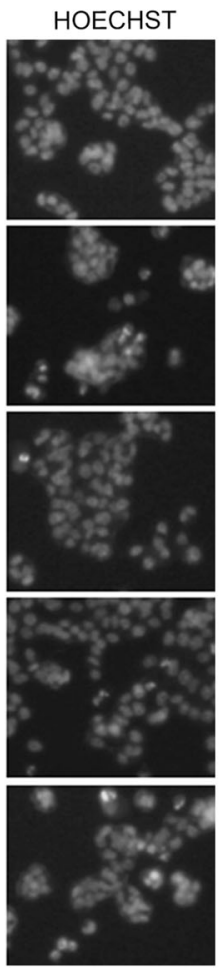

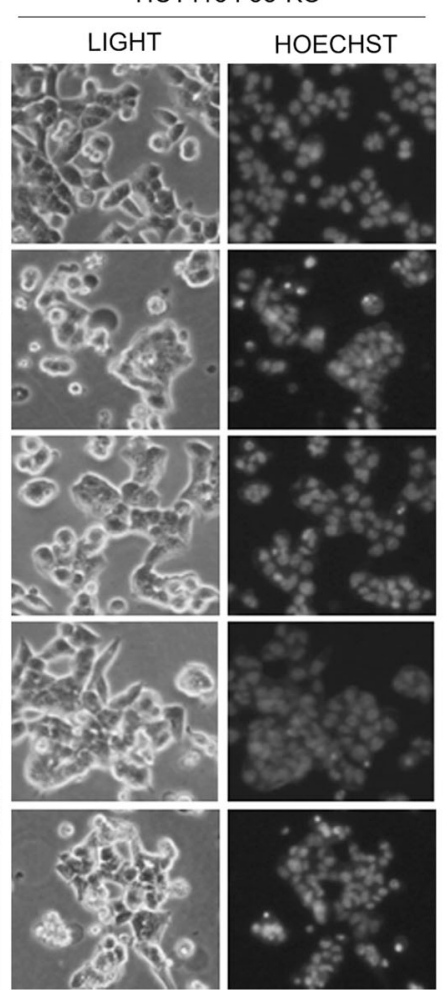

Apoptotic cells (\%)

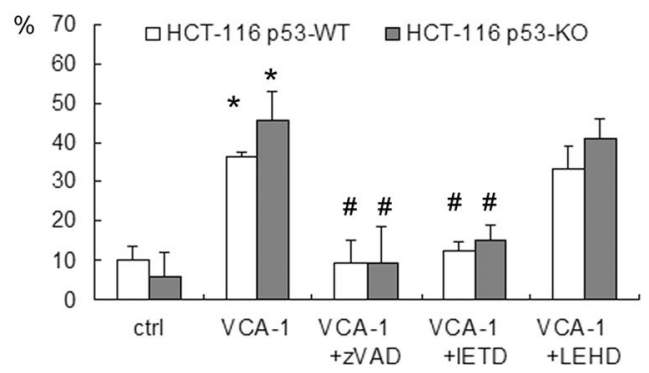

d

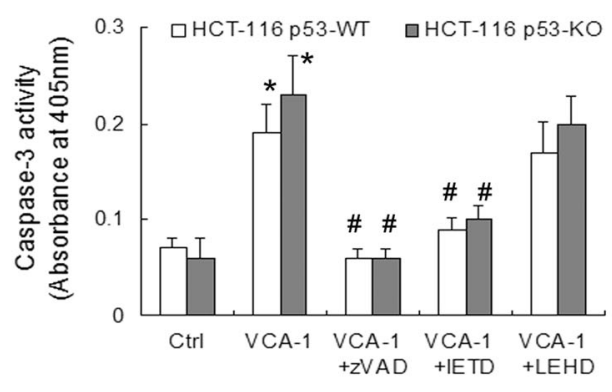

Fig. 3 VCA-1 induces caspase 8-dependent apoptosis in human cancer cell lines. a HCT116 p53-WT/KO cells were treated with VCA-1 (20 $\mu$ M) for the indicated periods. Cells were collected and subjected to Western blotting for the detection of caspases cleavage. $\mathbf{b}$ Inhibition of VCA-1induced PARP cleavage by pan-caspase inhibitor zVAD. HCT116 p53-WT/KO cells were pretreated with zVAD (20 $\mu \mathrm{M})$ for 45 min, followed with VCA-1 $(20 \mu \mathrm{M})$ treatment for $24 \mathrm{~h}$. PARP cleavage was detected by Western blotting. $\mathbf{c}$, $\mathbf{d}$ Inhibition of VCA-1-induced apoptosis by pan-caspase inhibitor ZVAD, caspase-8 specific inhibitor IETD-FMK or caspase-9 specific inhibitor LEHD-FMK. Cells were treated as same as described in Panel B. The proportions of cell death were determined by Hoechst staining (c) while the caspase-3 activities were measured colorimetrically at $405 \mathrm{~nm}(\mathbf{d}) .{ }^{*} P<0.05$, compared to the untreated control; ${ }^{\#} P<0.05$, compared to the VCA-1-treated cells

according to the manufacturer's protocol [14]. The Flag-XIAP expression vector was a generous gift from Dr. Colin Duckett (University of Michigan). The cotransfected marker pDsRed-N1 was purchased from Clontech (Palo Alto, CA, USA).

\section{Statistical analysis}

The experimental differences of the results were analyzed by SPSS software using one-way ANOVA or two-tailed $t$-test. The value of $P$ less than 0.05 were considered as statistically significant. 
a

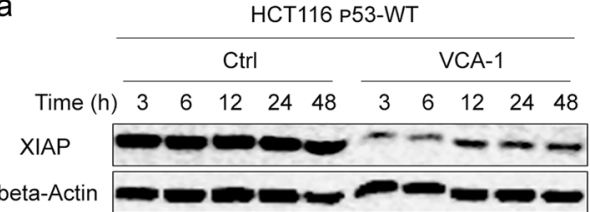

HCT-116 p53-WT

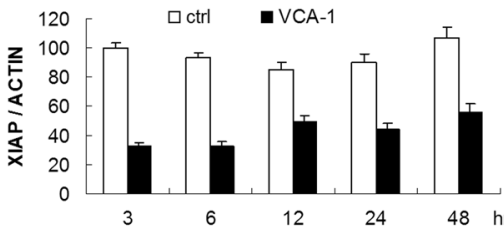

b

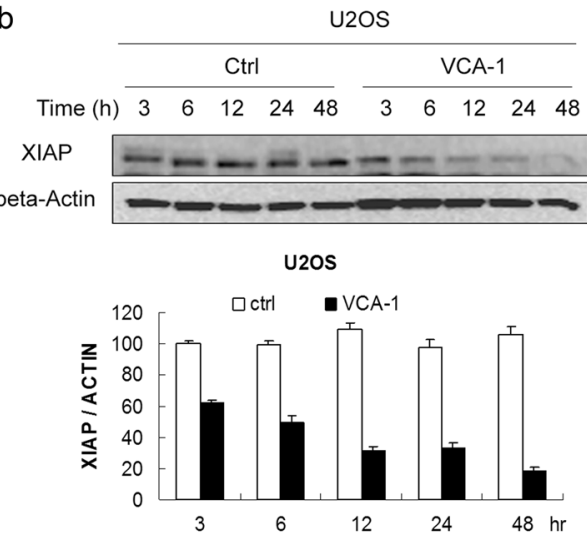

C
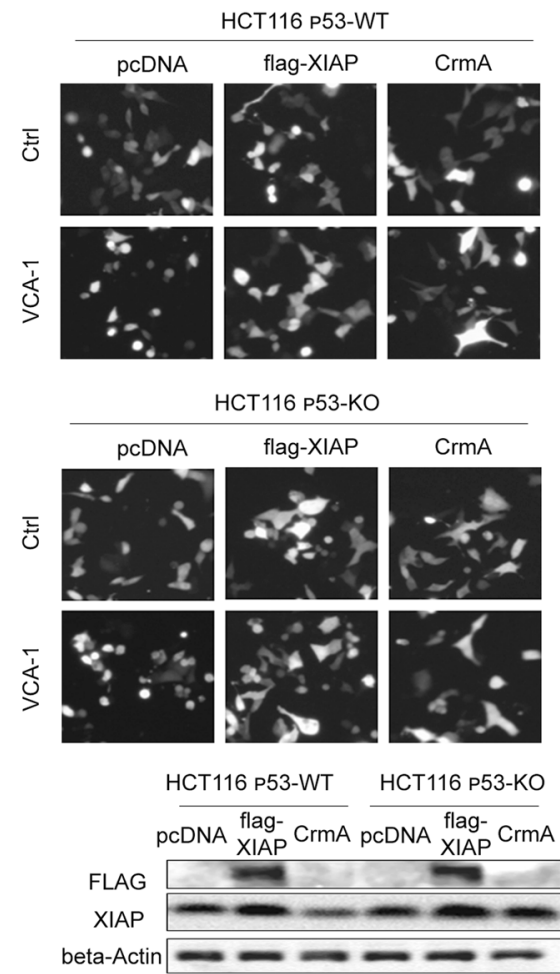
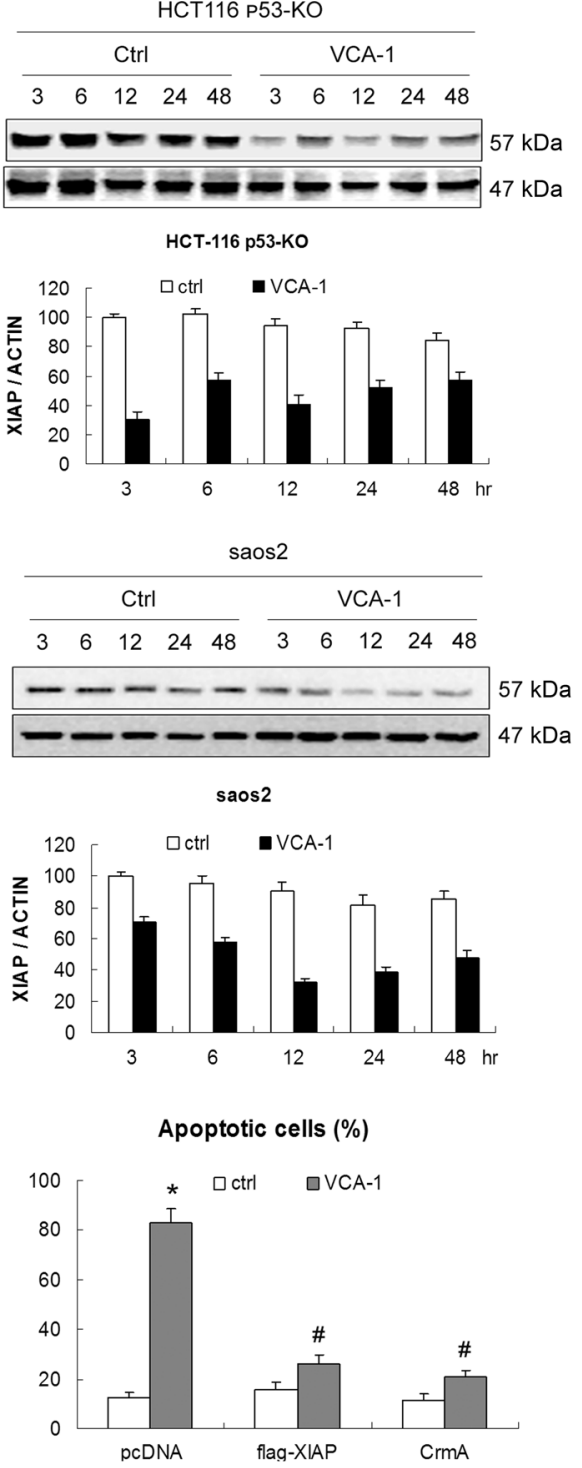

Apoptotic cells (\%)

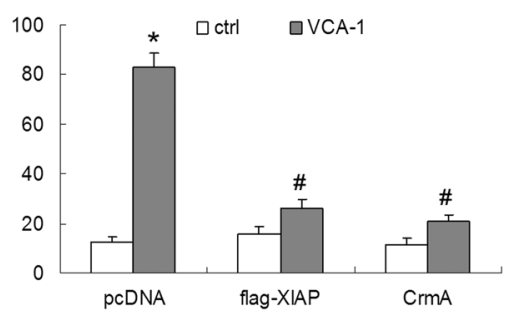

$58 \mathrm{kDa}$

$57 \mathrm{kDa}$

$47 \mathrm{kDa}$

Fig. 4 XIAP down regulation contributes to apoptosis induced by VCA-1. a, b HCT116 p53-WT/KO cells (a) and U2OS/Saos2 cells (b) were treated with VCA-1 $(20 \mu \mathrm{M})$ for the indicated time, and then cells were collected for detection of XIAP protein levels using Western blotting. C HCT116 p53-WT/KO cells were transiently transfected with pCDNA, Flag-XIAP, or CrmA, using pDsRed as a transfection marker. After transfection for $24 \mathrm{~h}$, cells were treated with VCA-1 $(20 \mu \mathrm{M})$ for another $24 \mathrm{~h}$. Cell death was then evaluated by morphological changes under a fluorescent microscope. Successfully transfected cells showed bright red color and those dead cells were identified by their morphological changes. The Western blotting assay showed the forced over-expression of flag-XIAP. ${ }^{*} P<0.05$, compared to the untreated control; ${ }^{\#} P<0.05$, compared to the VCA-1-treated cells 
cells was further validated using the sensitive $\gamma$-H2AX formation assay. As shown in Fig. 1C, VCA-1 induces $\gamma$-H2AX phosphorylation (ser 139) in a dose-dependent manner as a rapid DNA damage response.

To explore the potential anti-cancer effect of VCA-1, two pairs of p53 wild-type and p53 knockout human cancer cell lines, namely, human colorectal cancer cells HCT116 (p53-WT and KO) and human osteosarcoma p53-WT/KO cells-U2OS/Saos2, were treated with increasing concentrations of VCA-1 for $24 \mathrm{~h}$. Apoptotic cell death induced by VCA- 1 was determined by Hoechst staining according to the presence of typical nuclear condensation. As shown in Fig. 2a, b, the cells with or without p53 display similar responses to VCA-1 treatment. Representative apoptotic images after VCA-1 treatment are also presented in Fig. 2a, b lower panel. Consistent with the morphological results, PARP cleavages are also observed in all cell lines in both dose-dependent and time-dependent manners (Fig. 2c, d and data not shown), providing further evidences that VCA-1 is capable of inducing apoptosis independent of p53. In contrast, Andrographolide (a diterpenoid lactone) has been documented to induce apoptosis depending on the presence of functional p53 [14]. As shown in Fig. 2e, andrographolide treatment markedly cleaves PARP in HCT116 p53-WT cells, but not in HCT116 p53-KO cells.
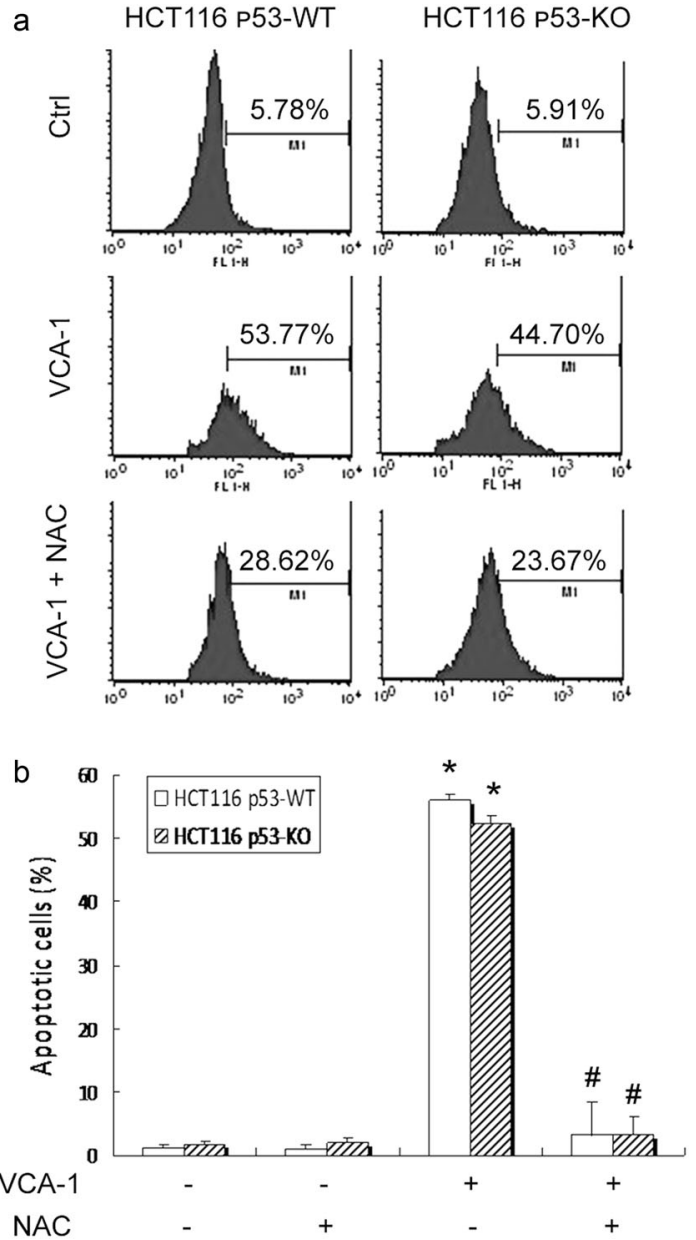

C

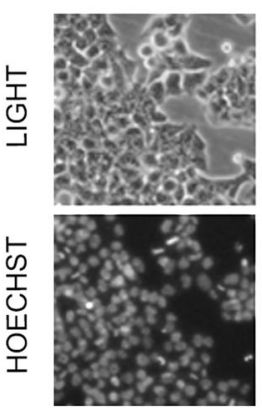

VCA-1

NAC
VCA-1 induces caspase-8-involved extrinsic apoptosis pathway Cellular apoptosis can be triggered extrinsically, intrinsically or both. The extrinsic cell death receptor pathway involves caspase-8 as the initiator in caspase cascade, while the intrinsic pathway involves caspase- 9 as the initiator. But both pathways end in caspase- 3 as the final executor in the cascade $[15,16]$. We thus tested VCA-1-induced caspase cascade in p53-WT/KO cancer cell lines. A time-dependent cleavage of caspase- 8 and caspase- 3 , rather than caspase-9 cleavage, are observable in VCA-1 treated HCT116 (p53-WT and -KO) cell lines (Fig. 3a). Next, we used a pancaspase inhibitor zVAD to both cell lines. As shown in Fig. 3b, $c$, zVAD is able to suppress PARP cleavage and apoptotic cell death induced by VCA-1 treatment. More importantly, caspase 8 specific inhibitor z-IETD-FMK, but not caspase 9 specific inhibitor z-LEHDFMK, could repress VCA-1 induced cell death. Consistently, the caspase-3 activity assay (Fig. $3 d$ ) could reproduce the result that zVAD and z-IETD-FMK, rather than z-LEHD-FMK reduce VCA-1 triggered induction of caspase- 3 activity. These results together strengthened the essential roles of caspase- 8 in cell death induced by VCA-1.

XIAP reduction is critical in VCA-1-induced apoptosis It has been well established that a group of anti-apoptotic proteins, such as FLIP, c-IAP, Bcl-2, and XIAP, are able to block 

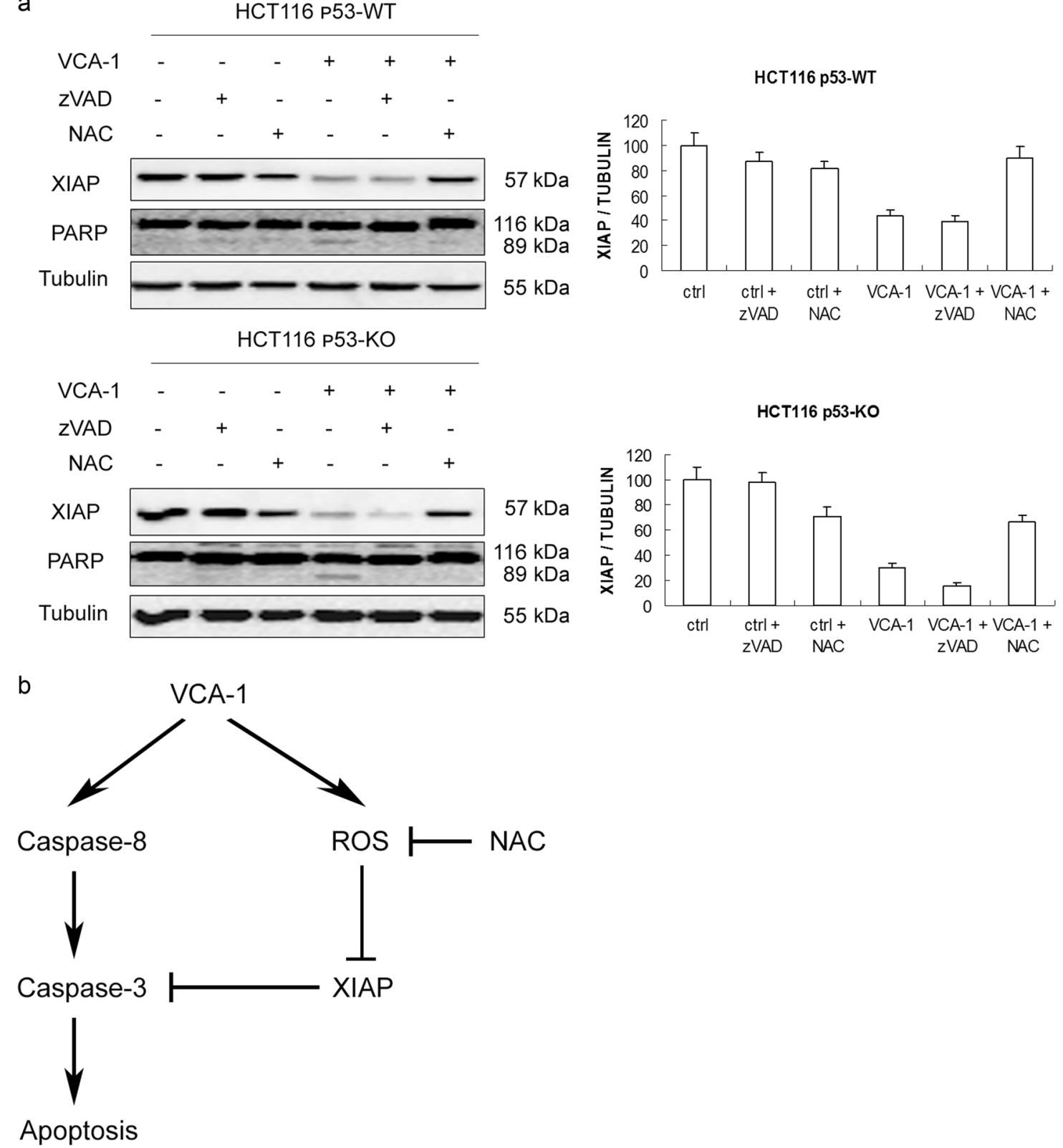

Fig. 6 Blockage of ROS reverses VCA-1-induced XIAP reduction. a HCT116 p53-WT/KO cells were pretreated with NAC (10 mM) or zVAD $(20 \mu \mathrm{M})$ as indicated for $45 \mathrm{~min}$ with or without VCA-1 $(20 \mu \mathrm{M})$ treatment for $12 \mathrm{~h}$. Cells were collected and subjected to Western blotting for detection of XIAP and PARP cleavage. b Illustration of possible pathways involved in VCA-1-induced apoptosis in cancer cells

apoptosis via inhibition of the caspase cascade [17-19]. To investigate the underlying mechanism of the apoptosis inducing function of VCA-1, we then tested changes of several antiapoptotic proteins in cells treated with VCA-1. While no significant changes were found for other proteins (data not shown), we observed evident reduction of XIAP protein level in all four cells lines tested (Fig. 4a, b). To determine the role of XIAP in VCA-1induced apoptosis, we over-expressed wild-type XIAP plasmids (Flag-XIAP) into HCT116 (p53-WT/KO) cells. The known viral protein CrmA was also used as a positive control [14, 20]. As shown in Fig. 4c, forced over-expression of XIAP offers significant protection against VCA-1-induced cell death, in comparison to those transfected with pcDNA vector control. The protective effect of XIAP is comparable to that of the known caspase inhibitor CrmA as a positive control (Fig. 4c). The above results demonstrate that XIAP reduction plays a crucial role in VCA-1-induced apoptosis.
VCA-1 enhances intracellular ROS production

Excess intracellular ROS are known to induce apoptosis [21, 22]. ROS generation has been suggested as a major anticancer mechanism by multiple chemotherapeutic agents [14, 23-25]. In particular, ROS and oxidative stress have been implicated in DNAdamage induced by varacin C [3]. We hence examined the intracellular ROS level in cells treated with VCA-1. As detected by flow cytometry, intracellular ROS increases following VCA-1 treatment in HCT116 p53-WT cells, as well as in p53-KO cells (Fig. $5 \mathrm{a}$ ), suggesting that VCA-1-mediated ROS production is likely to be p53-independent. Moreover, the ROS level in VCA-1treated cells is markedly reduced by NAC, a well-known antioxidant. More importantly, NAC protects cancer cells from VCA-1-induced apoptosis (Fig. 5b,c), suggesting that intracellular ROS level plays a critical role in VCA-1-induced apoptotic cell death. 
ROS contribute to XIAP downregulation in VAC-1-induced apoptosis

To understand the upstream regulator of XIAP reduction in VCA-1 treated cells, we further examined whether the enhanced ROS level contributes to the observed XIAP reduction in cells treated with VCA-1. Here, we compared the XIAP protein in HCT116 (p53WT/KO) cells with zVAD or NAC, respectively. As shown in Fig. 6a, NAC completely blocks XIAP reduction and PARP cleavage, thus indicating that ROS and oxidative stress contribute to $\mathrm{XIAP}$ reduction and consequent cell death. In contrast ZVAD, although rescues PARP cleavage, is unable to prevent XIAP reduction, suggesting that XIAP reduction is not due to caspasemediated cleavage. Based on the fact that zVAD successfully blocked VCA-1-induced apoptosis but not XIAP reduction, it is believed that XIAP may lies upstream of caspase activation. Taken together, as illustrated in Fig. $6 \mathrm{~b}$, data from this study suggest that VCA-1 induces apoptotic cell death via caspase activation and ROS-mediated XIAP down regulation, a process independent of $\mathrm{p} 53$.

\section{DISCUSSION}

The newly synthesized VCA-1 in our laboratory, as an analog of varacin $C$, has the benzotrithiol 1-oxide as its functionality. In this study we first reported that VCA-1 is capable of inducing apoptotic cell death in both human colorectal and osteosarcoma cells. More importantly, induction of apoptosis by VCA-1 is p53-independent. p53 is considered as the guardian of the genome due to its critical role in coordinating cellular responses to a board range of stress stimuli [6-8]. Consequently, cells without a functional p53 are known to be resistant to many chemotherapeutic agents [26, 27]. However, p53 mutation is found in approximately $50 \%$ of human cancer and those p53-negative cancers are usually with poor prognosis due to their poor response to therapeutic agents [12]. In this regard, therapeutic agents independent of p53 status held great advantages over those relying on p53 function in cancer chemotherapy. In the present study, we were able to prove VCA-1 exerts apoptosis inducing effect in both p53-WT/KO cell lines. Therefore, it is believed that the p53-independent function of VCA-1 makes it a promising candidate for a chemotherapeutic agent for cancer treatment.

Another important finding from this study is that ROS and oxidative stress play a critical role in VCA-1-mediated apoptosis, supported by the following two observations. Firstly, VCA-1 markedly enhanced the intracellular ROS level in both p53-WT and p53-KO cancer cells (Fig. 5a). Secondly, NAC, a wellestablished antioxidant, abolished VCA-1-induced ROS level and protected cells against apoptosis (Fig. 5b). The latter finding was consistent with the results of varacin $C$ [3], and believed to be associated with the trithiol 1-oxide as its reactive functionality. Many other chemotherapeutic drugs also have the same ROSdependent effects on induction of cell death [14, 23-25]. These results, taken together, reiterated the conclusion that cancer cells have imbalanced intracellular ROS levels with increased ROS generation and deregulated antioxidant system, making the cancer cells more susceptible to ROS-inducing drugs than the normal cells.

In the present study we observed that caspase-8, rather than caspase-9, was cleaved and activated after VCA-1 treatment (Fig. 3a). Pre-treatment with a specific caspase-9 inhibitor Z-LEHDFMK failed to protect cells from VCA-1-induced caspase-3 activation and apoptosis (Fig. 3c, d), further strengthened the importance of caspase-8 involved extrinsic apoptosis pathway in VAC-1 cytotoxicity. Among several antiapoptotic proteins, significant XIAP reduction was observed during VCA-1 treatment (Fig. 4a, b). Overexpression of XIAP effectively protected cancer cells from apoptosis, suggesting that XIAP downregulation plays a pivotal role in VCA-1-induced apoptosis (Fig. 4c). XIAP, a member of the inhibitor of apoptosis (IAP) family, is by far the most potent inhibitor of caspase- 3 and caspase- 9 [28], and therefore crucial to cell survival. In the exploration of the underlying mechanism for XIAP downregulation induced by VCA-1, we found that antioxidant NAC, but not the pan-caspase inhibitor ZVAD, was capable of completely reversing the XIAP reduction (Fig. 6a). It is thus clear that the elevated ROS level is responsible for XIAP downregulation and subsequent apoptosis. At present, it is not yet clear how ROS regulate XIAP protein level. It has recently been reported that XIAP reduces intracellular ROS accumulation by enhancing antioxidant gene expression $[29,30]$, which is inconsistent with the data in this study. It is probable that the coupling of XIAP and ROS generation is bidirectional, forming a positive feedback loop: VCA-1 stimulated-ROS production causes XIAP downregulation which in turn leads to a further increase in ROS generation. Obviously, the exact mechanisms for ROS-mediated XIAP down regulation remain to be further elucidated.

In conclusion, this study demonstrates for the first time that VCA-1, an analog of varacin C, is capable of inducing apoptosis in cancer cells, a process independent of p53. Excessive ROS generation is believed to play a fundamental role in VCA-1induced apoptosis, probably by XIAP reduction. Data from this study thus provide experimental evidence to support the further development of VCA-1 as a potential cancer chemotherapeutic agent, especially in p53-mutant cancers.

\section{ACKNOWLEDGEMENTS}

We thank Dr. Han-ming Shen (National University of Singapore) for his generous research guidance and technical advices, Dr. Vogelstein (Johns Hopkins University, Baltimore, MA, USA) for his kind gift of human HCT116 p53-WT/KO cells, Dr. Colin Duckett (University of Michigan) for the Flag-XIAP expression vector. This work was supported by the National Natural Science Foundation of China (31601120), the Guangxi Hundred-Talent Program (2014) and the Guangxi Natural Science Foundation (2015GXNSFAA139201) awarded to Jing Zhou; and by the Guangx Natural Science Foundation (2015GXNSFCB139004), the Guangxi Hundred-Talent Program (2016) and the Guangxi Medical University Training Program for Distinguished Young Scholars (2017) awarded to Guo-dong Lu.

\section{AUTHOR CONTRIBUTIONS}

J.Z. and W.L.L performed equally the majority of experiments and analyzed the data; Y.L. synthesized VCA-1 and other analogs; Z.X.W., Y.T., and X.X.H. participated in the literature review and statistical analysis; N.Y.C. and J.H.D. produced and analyzed the functional data; J. Z. and G.D.L. designed the research and wrote the paper.

\section{ADDITIONAL INFORMATION}

Competing interests: The authors declare no competing financial interests.

\section{REFERENCES}

1. Makarieva TN et al. Varacin and three new marine antimicrobial polysulfides from the far-eastern ascidian Polycitor sp. J Nat Prod. 1995;58:254-8.

2. Kulikov $A V$ et al. A new synthetic varacin analogue, 8-(trifluoromethyl)-1,2,3,4,5benzopentathiepin-6-amine hydrochloride (TC-2153), decreased hereditary catalepsy and increased the BDNF gene expression in the hippocampus in mice. Psychopharmacology. 2012;221:469-78.

3. Lee AHF et al. Acid-promoted DNA-cleaving activities and total synthesis of varacin C. J Am Chem Soc. 2002;124:13972-3.

4. De MA, Marino ML, Fais S. A rationale for the use of proton pump inhibitors as antineoplastic agents. Curr Pharm Des. 2012;18:1395-406.

5. Kanamala M, Wilson WR, Yang M, Palmer BD, Wu Z. Mechanisms and biomaterials in $\mathrm{pH}$-responsive tumour targeted drug delivery: a review. Biomaterials. 2016;85:152-67.

6. Carvajal LA, Manfredi JJ. Another fork in the road-life or death decisions by the tumour suppressor p53. EMBO Rep. 2013;14:414-21.

7. Speidel D. The role of DNA damage responses in p53 biology. Arch Toxicol 2015;89:501-17.

8. Bieging KT, Mello SS, Attardi LD. Unravelling mechanisms of p53-mediated tumour suppression. Nat Rev Cancer. 2014;14:359-70. 
9. Gurpinar E, Vousden KH. Hitting cancers' weak spots: vulnerabilities imposed by p53 mutation. Trends Cell Biol. 2015;25:486-95.

10. Kastenhuber ER, Lowe SW. Putting p53 in context. Cell. 2017;170:1062-78.

11. Muller PA et al. Mutant p53 drives invasion by promoting integrin recycling. Cell. 2009;139:1327-41.

12. Muller PA, Vousden $\mathrm{KH}$. Mutant p53 in cancer: new functions and therapeutic opportunities. Cancer Cell. 2014;25:304-17.

13. Lu GD et al. CCAAT/enhancer binding protein alpha predicts poorer prognosis and prevents energy starvation-induced cell death in hepatocellular carcinoma. Hepatology. 2015;61:965-78.

14. Zhou J, Lu GD, Ong CS, Ong CN, Shen HM. Andrographolide sensitizes cancer cells to TRAIL-induced apoptosis via p53-mediated death receptor 4 upregulation. Mol. Cancer Ther. 2008;7:2170-80.

15. Green DR, Llambi F. Cell death signaling. Cold Spring Harb. Perspect Biol. 2015;7: a006080.

16. Earnshaw WC, Martins LM, Kaufmann SH. Mammalian caspases: structure, activation, substrates, and functions during apoptosis. Annu Rev Biochem. 1999;68:383-424.

17. Kaufmann T, Strasser A, Jost PJ. Fas death receptor signalling: roles of Bid and XIAP. Cell Death Differ. 2012;19:42-50.

18. Roy N, Deveraux QL, Takahashi R, Salvesen GS, Reed JC. The C-IAP-1 and C-IAP-2 proteins are direct inhibitors of specific caspases. EMBO J. 1997;16:6914-25.

19. Wu H., Jeffrey Medeiros L., \& Young K. H. Apoptosis signaling and BCL-2 pathways provide opportunities for novel targeted therapeutic strategies in hematologic malignances. Blood Rev. https://doi.org/10.1016/j.blre.2017.08.004, 2017.

20. Wang $\mathrm{R}$ et al. Histone $\mathrm{H} 4$ expression is cooperatively maintained by IKKfS and Akt1 which attenuates cisplatin-induced apoptosis through the DNA-PK/RIP1/ IAPs signaling cascade. Sci Rep. 2017;7:41715.
21. Prasad S, Gupta SC, Tyagi AK. Reactive oxygen species (ROS) and cancer: Role of antioxidative nutraceuticals. Cancer Lett. 2017;387:95-105.

22. Galadari S, Rahman A, Pallichankandy S, Thayyullathil F. Reactive oxygen species and cancer paradox: to promote or to suppress? Free Radic Biol Med. 2017;104:144-64.

23. Huang $Q$ et al. Increased mitochondrial fission promotes autophagy and hepatocellular carcinoma cell survival through the ROS-modulated coordi-nated regulation of the NFKB and TP53 pathways. Autophagy. 2016;12:999-1014.

24. Trachootham D, Lu W, Ogasawara MA, Nilsa RD, Huang P. Redox regulation of cell survival. Antioxid Redox Signal. 2008;10:1343-74.

25. Lu GD, Shen HM, Chung MCM, Ong CN. Critical role of oxidative stress and sustained JNK activation in aloe-emodin-mediated apoptotic cell death in human hepatoma cells. Carcinogenesis. 2007;28:1937-45.

26. Weller M. Predicting response to cancer chemotherapy: the role ofp53. Cell Tissue Res. 1998;292:435-45.

27. Brosh $R$, Rotter V. When mutants gain new powers: news from the mutant p53 field. Nat Rev Cancer. 2009;9:701-13.

28. Resch U, Schichl YM, Sattler S, De MR. XIAP regulates intracellular ROS by enhancing antioxidant gene expression. Biochem Biophys Res Commun. 2008;375:156-61.

29. Evans MK, Sauer SJ, Nath S, Robinson TJ, Morse MA, Devi GR. X-linked inhibitor of apoptosis protein mediates tumor cell resistance to antibody-dependent cellular cytotoxicity. Cell Death Dis. 2016;7:e2073.

30. Foster DM, Stauffer SH, Stone MR, Gookin JL. Proteasome inhibition of pathologic shedding of enterocytes to defend barrier function requires $\mathrm{X}$-linked inhibitor of apoptosis protein and nuclear factor kB. Gastroenterology. 2012;143:133-44. 